\title{
COMPARISON OF TRAINED AND UNTRAINED NOVICE DRIVERS' GAZE BEHAVIOR IN RISKY AND NON-RISKY SCENARIOS
}

\author{
Anuj Pradhan, ${ }^{1}$ Alexander Pollatsek, ${ }^{2} \&$ Donald L. Fisher ${ }^{1}$ \\ ${ }^{1}$ Department of Mechanical and Industrial Engineering \\ ${ }^{2}$ Department of Psychology \\ University of Massachusetts \\ Amherst, MA, USA \\ E-mail: apradhan@ecs.umass.edu,pollatsek@psych.umass.edu, \\ fisher@ecs.umass.edu
}

\begin{abstract}
Summary: PC-based training programs have been developed that have been shown to improve novice drivers' hazard anticipation skills. Such programs give novice drivers information about particular driving situations (scenarios) where hidden threats could appear. We wanted to know whether this improvement in trained novice drivers' scanning skills was simply because the trained drivers were scanning more in general or, instead, were scanning more specifically in the scenarios in which potential threats could appear. In order to evaluate this question, we trained 11 novice drivers using a PC-based program and then compared their hazard anticipation performance on a driving simulator with the hazard anticipation performance of 11 untrained novice drivers. The drivers' eye movements were recorded for the duration of the drives. The glances of the drivers to the right (the correct response in most of the risky scenarios) were analyzed for each of the relevant risky scenarios and for stretches of non-risky situations. The trained drivers did look to the right $6.5 \%$ more in the non-risky situations than did the untrained drivers, although the difference was far from significant. However, the trained drivers looked to the right $32.7 \%$ more in the risky scenarios than in the non-risky situations, indicating they were discriminating quite well between the two situations. The untrained drivers also showed a smaller, but significant, discrimination between the risky scenarios and non-risky situations, as they looked to the right $18.9 \%$ more in the risky scenarios than in the non-risky stretches.
\end{abstract}

\section{INTRODUCTION}

Automobile crashes are the leading cause of death for newly-licensed teen drivers (Centers for Disease Control and Prevention, 2004). Moreover, the youngest licensed drivers are overinvolved in crashes, both fatal and nonfatal. This holds whether the index is the number of crashes per licensed driver or the number of crashes per vehicle mile. For example, based on data gathered in 2001 and 2002, the Insurance Institute of Highway Safety (2005) estimates that sixteen-year-old drivers are involved in 9.3 fatal crashes per 100 million vehicle miles, a rate almost ten times that of 50-54 year olds. In many states, over $25 \%$ of the 16 year olds are involved in a crash (Wisconsin Department of Transportation, 2001). The most critical period is the first six months right after the teen receives his or her solo license. The crash rate for the newly-licensed drivers decreases by a factor of five during this period (McCartt, Shabanova, and 
Leaf, 2003). This suggests that the problem is not plausibly only due to developmental immaturity.

Driver education programs are one attempt to address this problem. The first known driver education program was established in 1916 (National Highway Traffic Safety Administration (NHTSA), 1994, page 3). However, it was not until 1976 that a full-scale, controlled evaluation of driver education was undertaken in a suburb of Atlanta, Georgia. The results were disappointing, both in the short and long term (NHTSA, pp. 7-8). Perhaps not surprisingly, recent research suggests that standard driver education programs are not addressing the major sources of novice driver crashes. The most extensive information on the sources of novice driver crashes has been reported in a study in which McKnight and McKnight (2003) reviewed 2000 police accident reports: 1000 reports of crashes involving drivers 16 and 17 years old and 1000 reports of crashes involving drivers 18 and 19 years old (the reports do not include the date of licensure, so it is not possible to know precisely how many years of driving experience each cohort had on average). The 16 and 17 year olds were about three times as likely to be involved in a crash as the 18 and 19 year old drivers. In absolute terms, inferred failures to search ahead, to the side, or to the rear were implicated in $43 \%$ of the crashes (what we will refer to as failures of hazard anticipation). Inferred failures of attention were implicated in $23 \%$ of the crashes. Finally, novice drivers were more likely to drive too fast for the road conditions, especially on curves and slick surfaces (21\%). Note that alcohol was observed to be a factor in only $2 \%$ of the crashes and very high speeds (greater than $70 \mathrm{mph}$ ) in only $1 \%$ of the crashes among the 16 and 17 year olds, contrary to what might be more widely perceived as the major problems

The conclusion that standard driver education programs are not effectively addressing these various causes of novice driver crashes comes from recent studies on a driving simulator. These studies indicate just how great are the differences in the hazard anticipation skills of novice and experienced drivers. For example, consider a simulator study that analyzed the differences among older drivers' (24 drivers aged 60-75), younger drivers' (24 drivers aged 18-26) and learner's permit drivers' (24 drivers aged 16-17) ability to anticipate hazards and thereby potentially reduce their exposure to risks (Pradhan, Hammel, DeRamus, Pollatsek, Noyce, and Fisher, 2005). Each participant drove four experimental blocks, where each block contained four risky scenarios joined by one continuous section of roadway so that participants were exposed to a total of 16 different risky scenarios. Between the four scenarios in a block, the driver had to navigate varying roadway geometries, traffic patterns, and traffic signs and signals. These sections between scenarios were meant to represent the relatively easy, risk free, driving conditions that are a larger part of usual driving. Each block took about eight minutes to drive. A complete record of the position and velocity of the vehicle was obtained 60 times per second, as well as a complete record of the areas where the subjects were fixating (the point of gaze).

All 16 scenarios that were developed can be viewed on the web (www.ecs.umass.edu/hpl) by clicking on the Younger Driver button on the left side. Two example scenarios can make clear how the eye movements of the drivers in the simulator revealed how well they were selectively attending to areas of a scenario where information could be obtained that would reduce their likelihood of a crash. (1) First, consider what we will refer to as the Truck Crosswalk scenario (a, plan view). In that scenario, a truck is stopped on the side of the road in front of a marked midblock crosswalk in a suburban development. The participant driver is in the circled car in the 
figure and so cannot see potential pedestrians crossing in front of the truck. Thus, the driver should look to the right for a pedestrian and also should steer further to the left as he or she passes in front of the truck. What the driver actually saw on the simulator is displayed in $\mathrm{b}$ (the dashed cross represents the driver's point of gaze overlaid on the video; this was not present in what the driver actually saw). Note that the threat never materializes in any of the scenarios in order to prevent drivers from becoming hypervigilant. However, pedestrians and other vehicles are present in the scenarios, so the driver has every reason to suspect that threats could potentially appear. (2) Second, consider the Truck Left Turn scenario (c). Here the driver is approaching a four-way signalized intersection. There is a left-turn-only lane in which a truck is waiting to take a left turn. The truck blocks the driver's view of vehicles across the intersection in the opposing lane that could be taking a left in front of the truck across the path of the driver. Here, it is glances to the left that indicate whether the driver anticipates the potential hazard.

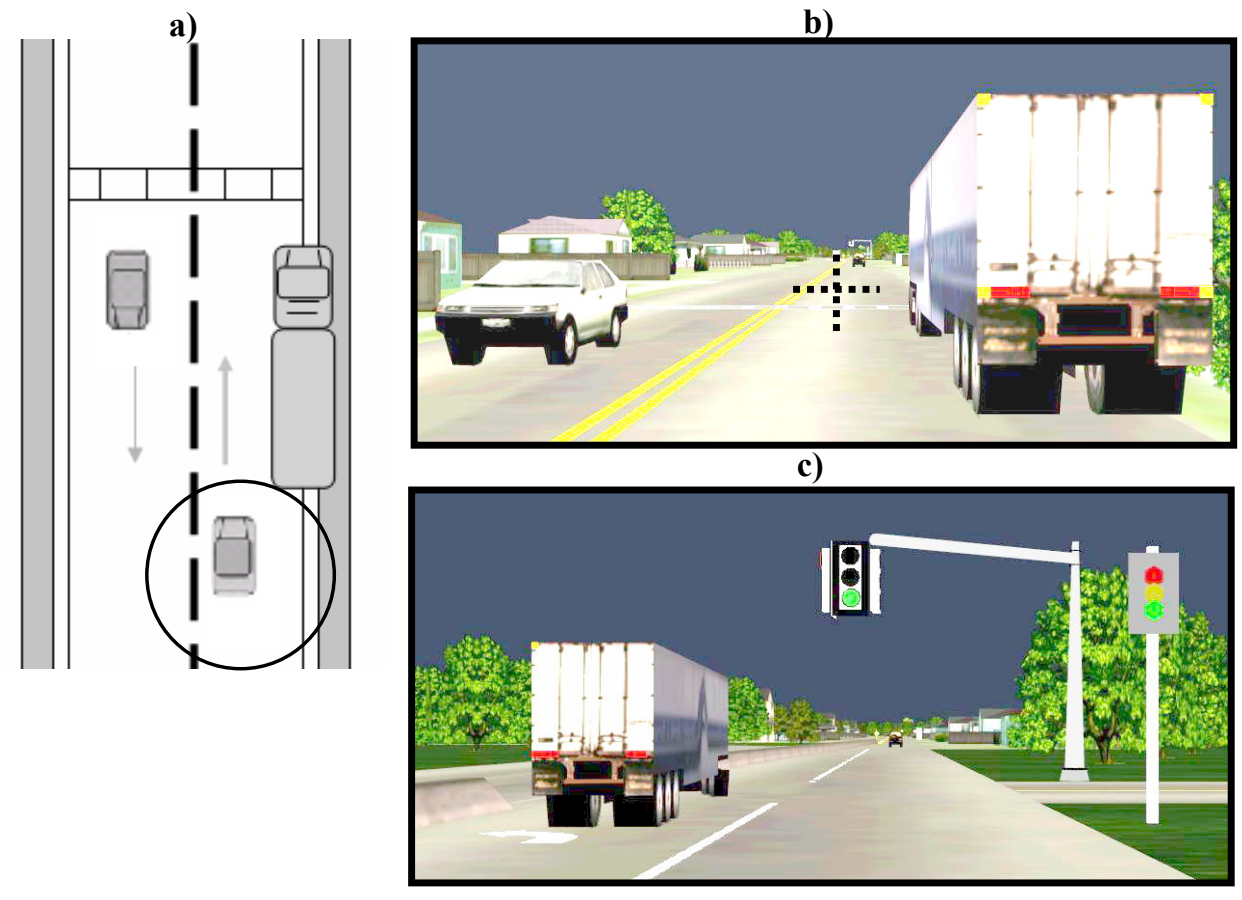

Figure 1. Truck Crosswalk Plan View; b) Truck Crosswalk Perspective View; c) Truck Left Turn Scenario

For the 14 scenarios that yielded interpretable results, there were 16 different measures of risk perception - a few including vehicle behaviors. Averaged over all 16 measures, the novice drivers engaged in behaviors indicative of their recognition of the potential for risk $35.1 \%$ of the time, the younger drivers engaged in such behaviors $50.3 \%$ of the time, and the older drivers, $66.2 \%$ of the time. The differences between each pair of cohorts were significant.

More recently, attempts have been made to determine whether novice drivers could be trained to anticipate hazards (Pollatsek, Narayanaan, Pradhan, and Fisher, 2006). Training clearly improved novice drivers' ability to anticipate hazards. We want to talk about an analysis of a recent study of the effects of hazard anticipation training on novice drivers that is very similar to the above study. Our original analyses (Pollatsek et al., 2006) showed that novice drivers could be trained to anticipate hazards better than untrained novice drivers and almost as well as 
experienced drivers. Specifically, trained novice drivers were more likely to scan in the area where a hidden threat might appear than were untrained novice drivers. However, we did not know whether this was because the training made the novice drivers more likely to scan all regions or, instead, the training influenced novice drivers only in the scenarios in which a threat was likely to appear. This is important to determine because novice drivers' resources are generally more limited than experienced drivers. Ideally, they would deploy their scanning efficiently and scan widely only when necessary.

\section{METHOD}

The details of the method used in this study have been presented in Pollatsek et al. (2006) and will only be given very briefly here.

\section{Participants}

Twenty-four novice drivers participated in the experiment. All were high school students who had had their learner's permit for 1 to 5 months. They were recruited from Amherst Regional High School and from driving schools in the Amherst area. Twelve were randomly assigned to the trained group and the other 12 to the untrained group. One participant from each group was eliminated in the analyses below because of some crucial missing data in the non-risky stretches.

\section{PC Training}

The Risk Awareness and Perception Training (RAPT) program relied primarily for explanation on plan views such as that displayed in a for the Truck Crosswalk scenario. A total of ten scenarios were presented to participants. In the pretest phase, participants were asked to use the mouse to drag red circles to areas of the roadway that should be continually monitored and, more importantly, drag yellow ovals to the areas that could contain information hidden from view but which is relevant to making a response that could reduce the driver's risks. For example, in the Truck Crosswalk scenario the participant should drag a yellow oval in front of the truck and a red circle immediately to the left of the front edge of the truck. After responding to a pretest scenario, the training phase for that scenario was initiated. The trainees were coached to appreciate where they should be looking for information that could reduce their risks. If the threat was caused by risks which were potentially hidden from view, the coaching involved both the use of diagrams to indicate what would be hidden from view and the pointing out of plausible risks such as pedestrians being obscured by the truck in the Truck Crosswalk scenario.

\section{Simulator Evaluation}

The evaluation of the training program was done using an advanced fixed-base driving simulator. This consists of a fully equipped 1995 Saturn sedan in front of which are three screens positioned to provide a $150^{\circ}$ horizontal field of view and $30^{\circ}$ vertical field of view (Figure 2). The virtual world is projected onto each of these screens with a resolution of $1024 \times 768$ pixels. A Silicon Graphics Infinite Reality Engine projects the graphical images at a rate of $60 \mathrm{~Hz}$. The software used to design and develop the virtual databases was Designers' Work Bench (Centric Software) and Real Drive Scenario Builder (Monterey Technologies). A driver can operate the controls of 
the vehicle just as in a real car on the open road, and would thus move through the simulated world accordingly.

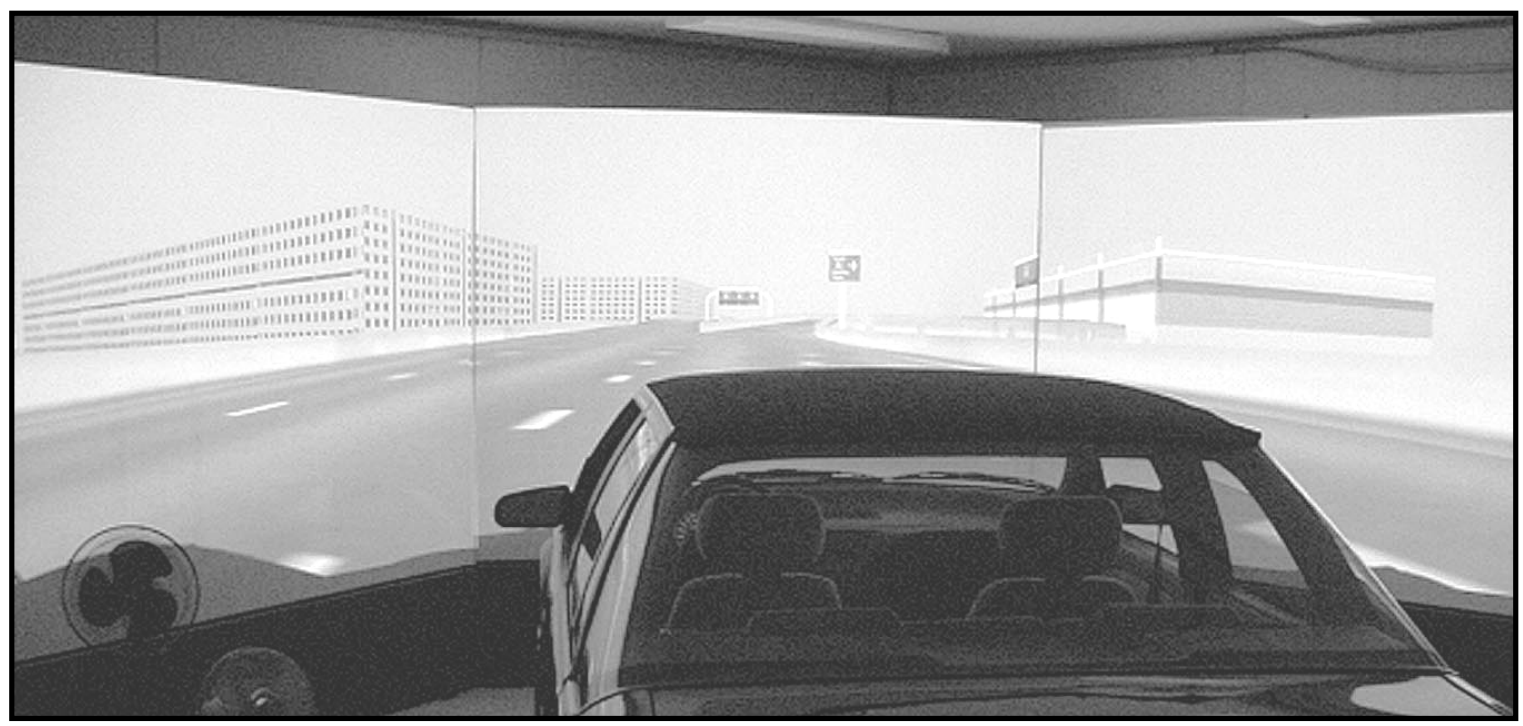

Figure 2. Driving Simulator

\section{Eye Tracker}

Each participant was fitted with an ASL5000 Series head-mounted eye tracker integrated with a magnetic head tracker. A direct output of the simulated images on the three screens was obtained from a different channel of the graphics engine and was used as the remote scene camera. The eye position of the participant was converted to external point of gaze and superimposed as crosshairs on the output of the remote scene camera at $60 \mathrm{~Hz}$, thus providing a real-time video of the drives with crosshairs superimposed where the driver was fixating at each moment.

\section{RESULTS}

Of key interest for the risky scenarios was whether the drivers scanned a region containing a potential risk. Similar to the results reported previously (e.g., Pollatsek et al., 2006), the trained drivers scanned the risk-relevant areas $23.6 \%$ more than the untrained drivers $(53.2 \%$ vs. $29.6 \%)$, $\mathrm{t}(20)=2.89, \mathrm{p}<.01$. The central issue that this study addressed is whether this difference was merely due to the trained drivers looking around more in general or whether, instead, it was due to their having learned to look at specific areas of risk. Because most of the risky scenarios involved a rightward look for the risk-relevant information, the most relevant comparison was the percentage of rightward glances in the risky scenarios for which a rightward glance captured the risk-relevant information with the percentage of glances to the right in the baseline non-risky stretches for which there was no particular reason to look in that direction. The trained drivers did look to the right in the non-risky stretches a bit more than the untrained drivers $(24.7 \%$ vs. $18.2 \%$; Table 1), although the difference was far from significant $(\mathrm{t}<1)$. However, it is clear that the trained drivers looked to the right far more in the risky scenarios than in the non-risky stretches $(57.4 \%$ vs. $24.7 \%), t(10)=4.44, p<.002$. The control group also looked significantly more to the right in the risky scenarios than in the non-risky stretches $(37.1 \%$ vs. $18.2 \%), t(10)=$ 
$2.49, \mathrm{p}<.05$. Obviously, the increase in rightward glances in the risky scenarios over the nonrisky stretches was bigger for the trained drivers than the untrained drivers $(32.7 \%$ vs. 18.9\%); however, this difference was only marginally significant $[\mathrm{t}(20)=1.31]$.

Table 1. Percent of Right Looks on Selected Risky Scenarios for which Right Look was Appropriate vs. Percent of right looks in non-risky stretches

\begin{tabular}{|c|c|c|c|}
\hline \multirow{2}{*}{ Group } & \multicolumn{2}{|c|}{ Scenarios } & \\
\cline { 2 - 4 } & Risky & Non-Risky & Difference \\
\hline Trained & $57.4 \%$ & $24.7 \%$ & $32.7 \%$ \\
\hline Untrained & $37.1 \%$ & $18.2 \%$ & $18.9 \%$ \\
\hline Difference & $20.3 \%$ & $6.5 \%$ & $13.8 \%$ \\
\hline
\end{tabular}

\section{DISCUSSION}

The above results indicate that the effects of the Risk Awareness Training Program are primarily what were intended: to train beginning drivers to glance away from directly in front of them at specific locations that were relevant to upcoming risks. The finding that trained drivers glanced at these locations more frequently than untrained drivers was first reported in Pollatsek et al. (2006). What is new here is the finding that there was some indication that the training also caused the trained drivers to look away from directly in front of them in stretches in which there was no particular indication of a potential risk, although this tendency was small and not significant. What is also new here, and of more importance, is the finding that the likelihood that the trained drivers glance to the right toward a potential threat when such existed was much larger $(32.7 \%)$ than the likelihood that they glanced to the right when no threat appeared. The untrained drivers also glanced to the right when a potential threat was present more often than when such a threat was not present (18.9\%), indicating that their rightward glances in the risky scenarios were not "random" but demonstrated some appreciation of the potential risks, though not as great as the trained drivers. Most importantly, the results indicate that our training was teaching the drivers to look to the right when it was important rather than just looking to the right more in general.

\section{REFERENCES}

Centers for Disease Control and Prevention. (2004). U.S. Motor vehicle injury facts. Available at http://www.cdc.gov/ncipc/whd2004/information/MV-Facts.pdf. Accessed May 16, 2006.

Insurance Institute for Highway Safety. (2005). Fatality facts 2005: Older people. Available at http://www.iihs.org/research/fatality_facts/olderpeople.html. Accessed May 8, 2007.

McCartt, A.T., Shabanova, V.I., and Leaf, W.A. (2003). Driving experience, crashes and traffic citations of teenage beginning drivers. Accident Analysis and Prevention, 35, 311-320. 
McKnight, J.A., and McKnight, S.A. (2003). Young novice drivers: Careless or clueless. Accident Analysis and Prevention, 35, 921-925.

National Highway Traffic Safety Administration. (July 2006). Traffic safety facts: Crashes stats: 16-year-ols have highest crash involvement among young drivers. (DOT HS 510 896). Accessed at www-nrd.nhtsa.dot.gov/pdf/nrd-30/NCSA/RNotes/2006/810596.pdf. Accessed August 24, 2006.

Pollatsek, A., Narayanaan, V., Pradhan, A.K., and Fisher, D.L. (2006). The Use of Eye Movements to Evaluate a PC-Based Risk Awareness and Perception Training (RAPT) Program on an Advanced Driving Simulator. Human Factors, 48, 447-464.

Pradhan, A.K., Hammel, K.R., DeRamus, R., Pollatsek, A., Noyce, D.A. and Fisher, D.L. (2005). The Use of Eye Movements to Evaluate the Effects of Driver Age on Risk Perception in an Advanced Driving Simulator. Human Factors, 47, 840-852.

Wisconsin Department of Transportation. (2001). Wisconsin crash facts on the 16-19 year old age group. Available at http://www.dot.wisconsin.gov/safety/motorist/crashfacts/docs/ archive/teenfacts2000.pdf. Accessed May 8, 2006. 